\title{
Rhodamine 6G conjugated to gold nanoparticles as labels for both SERS and fluorescence studies on live endothelial cells
}

\author{
Aleksandra Jaworska • Tomasz Wojcik • Kamilla Malek • \\ Urszula Kwolek • Mariusz Kepczynski • Abu A. Ansary • \\ Stefan Chlopicki • Malgorzata Baranska
}

Received: 14 April 2014 / Accepted: 28 May 2014 / Published online: 19 June 2014

(C) The Author(s) 2014. This article is published with open access at Springerlink.com

\begin{abstract}
Fluorescence and surface-enhanced Raman scattering (SERS) spectroscopy were employed to investigate the cellular uptake of rhodamine $6 \mathrm{G}(\mathrm{R} 6 \mathrm{G})$ alone and of R6G loaded with gold nanoparticles (AuNPs) by endothelial cells. R6G plays the role of a Raman reporter in SERS but also displays strong fluorescence. The presence of bare R6G molecules and R6G-AuNPs in the cytoplasm of the cells is detected via the 2D fluorescence of the dye after a $0.5 \mathrm{~h}$ of the incubation with R6G and R6G-AuNPs, and then the concentration of the dye increases within $4 \mathrm{~h}$ of exposure. The examination of the cellular uptake of the R6G and R6GAuNPs species at different temperatures suggests that the internalization of the R6G-AuNPs into endothelial cells occurs mainly via endocytosis. 3D fluorescence imaging of R6G inside cells reveals inhomogeneous distribution of the dye in the cytoplasm. The SERS signal of the Raman reporter inside the cell disappears after $2 \mathrm{~h}$ of incubation with R6G-AuNPs and then amino acid residues, purines and pyrimidines become SERS-active via their interactions with the gold. The results highlight the significance of using multiple techniques
\end{abstract}

Electronic supplementary material The online version of this article (doi:10.1007/s00604-014-1307-5) contains supplementary material, which is available to authorized users.

A. Jaworska $\cdot$ T. Wojcik $\cdot$ K. Malek $(\bowtie) \cdot$ M. Kepczynski $\cdot$

S. Chlopicki $\cdot$ M. Baranska

Jagiellonian Centre for Experimental Therapeutics (JCET),

Jagiellonian University, Bobrzynskiego 14, 30-348 Krakow, Poland

e-mail: malek@chemia.uj.edu.pl

A. Jaworska $\cdot$ K. Malek $\cdot$ U. Kwolek $\cdot$ M. Kepczynski $\cdot$

A. A. Ansary $\cdot$ M. Baranska

Faculty of Chemistry, Jagiellonian University, Ingardena 3,

30-060 Krakow, Poland

S. Chlopicki

Department of Experimental Pharmacology, Jagiellonian University

Medical College, Grzegorzecka 16, 31-531 Krakow, Poland to cover a spectrum of issues in the application of SERS nanosensors for probing an intracellular environment under comparable and standardized conditions.

Keywords Endothelium $\cdot$ SERS imaging $\cdot 2 \mathrm{D}$ and 3D fluorescence $\cdot$ Rhodamine $6 \mathrm{G} \cdot$ Cellular uptake

\section{Introduction}

Raman spectroscopy is a method that enables vibrational modes of individual bonds to be probed optically and has been widely employed in various biomedical experiments $[1,2]$. While the Raman scattering is a weak effect challenging to measure spectra from an ultra-low concentration of a sample, for molecules adsorbed on roughened metal surfaces of silver and gold it can be enhanced by up to 14 orders of magnitude by means of surface-enhanced Raman spectroscopy (SERS). This even includes single molecule detection [3, 4]. As a result, Raman cross-sections of molecules interacting with SERS-active substrates can reach values comparable to those observed for the fluorescence phenomenon [3]. SERS has been dynamically developing, especially in the field of bioanalysis, due to its ultrasensitive detection limits $[5,6]$. Moreover, informative SERS signals from the intracellular microenvironment can be collected within seconds in contrast to normal Raman scattering [7]. Gold-supported in vitro SERS in living cells provides a tool for sensitive and structurallyselective detection of native chemicals such as DNA, proteins, lipids etc., and for monitoring their intracellular distributions. However, the distribution of bare metal nanoparticles in the cells cannot be controlled [7-9]. Another application of SERS for cellular studies is based on nanoprobes conjugated to Raman reporters [5,8-11]. The most commonly used 
reporters are organic dyes, e.g. malachite green, rhodamine B or crystal violet [5-9].

On the other hand, nowadays confocal laser-scanning microscopy (CLSM) is a widely used technique for cellular imaging and biomedical diagnostics $[12,13]$. FluorescenceCLSM is the most appropriate method of imaging cells and subcellular structures, both in vitro and in vivo, once a cell shows intrinsic fluorescence or is labelled with a fluorescent probe [14]. In comparison to the conventional fluorescence microscopy, CLSM reduces the effect of light scattering enabling the observation of thick and light-scattering objects with a good quality $[13,15]$. Depending on the numerical aperture of the objective, the excitation wavelength, and the medium refraction index, the spatial resolution in the lateral and axial directions can be as much as $250 \mathrm{~nm}$ and $>600 \mathrm{~nm}$, respectively [13-15]. Furthermore, this microscopic technique allows the reconstruction of the original threedimensional structure of the objects in the specimen [13, 16]. As a consequence, CLSM in fluorescence mode provides information on distances between specific structures in the cells and enables imaging of cellular compartments [16] and monitoring physiological parameters and cellular metabolism [12]. Despite the obvious advantages, CLSM has several limitations such as photodamage and photobleaching of the sample $[12,14,15]$.

In this work both SERS and fluorescence microscopy are employed to study intracellular environment of endothelial cells. The endothelium is a monolayer of cells lining of all blood vessels (arteries, veins and capillaries) and the lymphatic system, and thus this is in a direct contact with blood/lymph and circulating in blood cells [17]. Currently, this type of the cells is recognized to be a predominant player in the control of blood fluidity, platelet aggregation, thrombosis, vascular tone. Endothelium regulates also immune response, inflammation and angiogenesis [18]. Therefore, endothelial dysfunction, a term that encompasses multiple potential defects of the endothelial cells, can tip the balance toward thrombosis and in consequence this contributes to various pathological states such as atherothrombosis, arterial thrombosis (stroke, visceral and peripheral artery occlusive diseases) and thrombotic microangiopathies [17]. Taking into consideration the importance of endothelium in the regulation of cardiovascular system, novel tools seem mandatory to foster studies on biochemistry and function of endothelial cells.

However, a major challenge of cellular studies in terms of SERS is the delivery of target nanoparticles in sufficient quantities to acquire a spectral signal allowing for simultaneous spatial distribution of the nanoparticle (through a reporter signal) and chemical information of the cellular environment (via the enhancement of Raman signal of biomolecules on a metallic support). Therefore, the SERS- and fluorescence-based studies on the uptake of rhodamine $6 \mathrm{G}$ conjugated to gold nanoparticles (R6G-AuNPs) by live endothelial cells have been undertaken in our laboratory. Rhodamine $6 \mathrm{G}$ is a fluorophore of a high cross-section for Raman scattering used by us before in cellular studies of macrophages and endothelial cells $[8,9]$. For in vitro culture we chose EA.hy926 cells, a line derived from human umbilical vein endothelial cells. Our preliminary investigations have shown variation in SERS information on biocomponents in this cell line along with elongation of incubation time [8]. Thus, we continue our SERS studies with a support of fluorescence microscopy since rhodamine $6 \mathrm{G}$ also exhibits a high crosssection for the fluorescence phenomenon. This allows us to follow cellular uptake of R6G-AuNPs and distribution of the Raman reporter inside the cell. Finally, we demonstrate a potential application of this label for probing live endothelial cells.

\section{Materials and methods}

\section{Materials}

Rhodamine 6G (R6G), gold (III) chloride trihydrate, sodium citrate tribasic dihydrate and paraformaldehyde were purchased from Sigma (www.sigmaaldrich.com) and were of analytical grade.

An aqueous stock solution of rhodamine $6 \mathrm{G}$ at the concentration of $10^{-3} \mathrm{M}$ was prepared by diluting an appropriate amount of the solid in the 4-fold distilled water. Gold nanoparticles (AuNPs) were prepared according to the Frens procedure [19]. In this synthesis Au ions are reduced by citrate ions. R6G-AuNPs solution was prepared by mixing $10 \mu \mathrm{L}$ of the $1 \times 10^{-3} \mathrm{M}$ solution of $\mathrm{R} 6 \mathrm{G}$ with $1 \mathrm{~mL}$ of the gold colloid.

\section{Cells and protocol of SERS and fluorescence experiment}

Experiments were conducted for EA.hy926 cells, a line derived from human umbilical vein endothelial cells fused with the A549 line [20]. Cells were grown in 6-well plates in DMEM supplemented with $10 \%$ fetal bovine serum, $2 \mathrm{mM}$ L-glutamine, penicillin, streptomycin, and $2 \%$ HAT. After $24 \mathrm{~h}$ passaging a medium was replaced by a medium containing solution of R6G-AuNPs. After $5 \mathrm{~min}$, medium was mixed with the R6G-AuNPs solution $(10: 1 \mathrm{v} / \mathrm{v})$. The final concentration of R6G and AuNPs in medium was $1 \times 10^{-6}$ and $1 \times 10^{-11} \mathrm{M}$, respectively. Cells were incubated in the medium containing R6G-AuNPs for $0.5,1,2,4$ and $16 \mathrm{~h}$. Two control experiments were carried out to exclude autofluorescence of cells and to exclude the contribution of normal Raman signal of the dye non-conjugated to AuNPs. Cells were incubated in a pure medium and a medium containing a pure R6G solution $\left(\mathrm{c}=1 \times 10^{-6} \mathrm{M}\right)$, respectively. Then, the medium was washed out three times with DPBS (Dulbeccos's phosphate-buffered saline) and cells were kept in a pure medium in an incubator until SERS and 2D fluorescence measurements were 
performed. For 3D fluorescence measurements, cells were fixed with $4 \%$ paraformaldehyde for $6 \mathrm{~min}$ and store in a fridge until measurements. For the all fluorescence measurements, nuclei were additionally stained with Hoechst 33342 [21].

Cell metabolic activity by the MTT assay

Cells were exposed to R6G and R6G-AuNPs for 0.5, 1, 2, 4, and $16 \mathrm{~h}$. Then, the medium was replaced by a $2 \mathrm{~mL}$ medium containing $0.5 \mathrm{mg} \cdot \mathrm{mL}^{-1}$ of 3-(4,5-dimethyl-2-thiazolyl)-2,5diphenyl-2H-tetrazolium bromide (MTT) followed by incubation cells for $3 \mathrm{~h}$ at $37^{\circ} \mathrm{C}$. Afterwards, the medium was carefully removed from the wells. DMSO:ethanol $(1: 1)$ was added to each well and the plate was shaked on a plate shaker for $5 \mathrm{~min}$ to dissolve formazan crystals. Absorbance of formazan was measured at $560 \mathrm{~nm}$ by a Biotec Synergy ${ }^{\mathrm{TM}} 4$ plate reader (www.biotek.com).

Instrumentation

The electronic absorption spectra of the gold colloid, the R6G solution and the gold colloid mixed with R6G were recorded with a UV-vis-NIR Perkin Elmer spectrophotometer (model Lambda 35) (www.perkinelmer.com) in the range of 190$1,100 \mathrm{~nm}$ with a resolution of $2 \mathrm{~nm}$. Quartz cells of $1 \mathrm{~cm}$ were used.

Fluorescence images of cells for the quantitative analysis of fluorescence were collected using Olympus $\mathrm{Scan}^{\wedge} \mathrm{R}$ system (www.olympus-europa.com) in DAPI and Cy3 channels for Hoechst 33342 and rhodamine 6G, respectively. Images were constructed by using Columbus data storage and analysis system (Perkin Elmer). 3D fluorescence images of R6GAuNPs-incubated cells were acquired with a A1-Si Nikon (Japan) confocal laser scanning system built onto a Nikon inverted microscope Ti-E using a Plan Apo $100 \times / 1.4$ Oil DIC objective (www.nikon.com). Images were recorded at a resolution of $1,024 \times 1,024$ while Hoechst 33342 and R6G were excited with 405 and $561 \mathrm{~nm}$ diode lasers, respectively. 3D fluorescence images were constructed by using a NISElements AR 3.2 software.

SERS mapping of cells and SERS spectra of R6Gconjugated AuNPs were carried out by using a WITec system (www.witec.de), equipped with an immersive objective with magnification of $60 \times$ and a He-Ne laser $(632.8 \mathrm{~nm})$. This optical setting provides max. spatial resolution of $0.4 \mu \mathrm{m}$. For all measurements, integration time was $0.05 \mathrm{~s}$ with a single accumulation, laser power of $10 \mathrm{~mW}$ and grating $600 \mathrm{~g} \cdot \mathrm{mm}^{-1}$. The recorded spectral range for this grating is $0-2,400 \mathrm{~cm}^{-1}$. SERS spectra of R6G-AuNPs as a reference for cells mapping were recorded by placing a sample in a glass cuvette and using an air objective (20×). Three SERS spectra were acquired, each for freshly prepared sample. The latter was prepared by mixing $500 \mu \mathrm{L}$ of the Au colloid with $5 \mu \mathrm{L}$ of a $1 \times 10^{-3} \mathrm{M}$ R6G solution in a glass cuvette. For Raman mapping, raster scans over single living cells were carried out with a computercontrolled $x, y$-stage. The mapping step was $1 \mu \mathrm{m}$.

\section{Analysis of SERS maps}

Hierarchical K-means cluster analysis of SERS images was performed by using a WITec Project 2.06 software. The spectra were analysed in the region of $200-1,800 \mathrm{~cm}^{-1}$ after a routine procedure for cosmic rays removal and smoothing (13 points) using a Savitzky-Golay algorithm.

\section{Results and discussion}

Spectral characterisation of R6G-AuNPs

An important step in the synthesis of SERS labels is selection of an appropriate metal surface. The metallic support should be stable, small enough to easily cross the cellular membrane, and cause a relatively low cytotoxicity. In our work gold nanoparticles with the average diameter of $40 \mathrm{~nm}$ were chosen. The DLS (Dynamic Light Scattering) method confirmed the size of AuNPs (approx. $45 \mathrm{~nm}$ ) while AFM images showed clearly the spherical shape of non-aggregated nanoparticles (Fig. S1 in Electronic Supporting Material, ESM). According to DLS, gold nanoparticles exhibited relatively low polydispersity index of 0.38 . The UV-vis spectra display the presence of a LSPR band of the colloid at $527 \mathrm{~nm}$, typical for this diameter of the Au sol (Fig. S2, ESM). Then a spectrum of the dye mixed with the gold colloid shows the characteristics of aggregate formation by the presence of extended plasmon band with a second maximum at $641 \mathrm{~nm}, c$.f. Fig S2. Formation of small aggregates results in an increase of SERS enhancement factors [22]. Here, the chosen size of AuNPs and concentration of R6G provide a stable SERS signal of the dye. Figure S3 (ESM) shows the comparison of the exemplary three raw SERS spectra of R6G adsorbed on the gold colloid used further in SERS mapping of cells. Positions, full width at half maximum and relative intensities of SERS bands are identical in each spectrum while the total intensity of the spectra in the recorded region varies within $c a .7 \%$. This confirms the stability of the SERS signal of the Raman reporter resulting from a relatively similar aggregation of the gold nanoparticles.

\section{Cytotoxicity of R6G and R6G-AuNPs}

A cytotoxic effect of the gold and silver nanoparticles has been previously reported [23, 24]. The exposition of $\mathrm{C} 17.2$ neural progenitor, human umbilical vein endothelial, and PC12 rat pheochromocytoma cells to AuNPs at the 
concentration of $100 \mathrm{nM}$ for $24 \mathrm{~h}$ did not induce acute cytotoxicity as activity of lactate dehydrogenase (LDH) revealed while deformation of actin and tubulin cytoskeleton was observed for $50 \mathrm{nM}$ concentration of AuNPs [24]. However, this effect is time- and concentration-dependent as well as it can be specific for a type of cells. In our work, we used a lower concentration of AuNPs (10 pM in medium) than in the literature [24], therefore we excluded a toxic influence of the gold nanoparticles on the EA.hy926 cells. Next, we evaluated toxicity of R6G and R6G-AuNPs by performing the MTT assay (Table 1). The MTT assay, which is sensitive to mitochondrial activity, exhibits not only cell apoptosis but also the inhibition of cell division. The EA.hy926 cells are sensitive to rhodamine $6 \mathrm{G}$ after long incubation time whereas the presence of both the dye and the gold nanoparticles affects the cells even after 1-h contact. Non-toxic effect of the SERS label is observed up to $4 \mathrm{~h}$ and then massive apoptosis/necrosis of the endothelial cells takes place after $16 \mathrm{~h}$ of incubation. This indicates that EA.hy926 cells can be exposed to nanocarries in a relatively short time of a few hours.

The uptake of R6G and R6G-AuNPs by endothelial cells: 2D quantitative intracellular fluorescence

A crucial step in the application of the dyes and nanoparticles in detection of intracellular chemical environment is determination of the uptake mechanism of non-conjugated and conjugated dye molecules to Au nanoparticles. For this purpose $2 \mathrm{D}$ quantitative fluorescence spectroscopy can be used to determine an amount of a labile fluorescent reporter inside cells. However, such information cannot be achieved for the bare metal nanoparticles as they do not possess the fluorescent features. In our approach, we employed incubation time from 0.5 till $16 \mathrm{~h}(0.5,1,2,4$ and $16 \mathrm{~h}$ ) keeping cells in an optimal temperature of $37^{\circ} \mathrm{C}$ as well as under energy depletion conditions at 25 and $4{ }^{\circ} \mathrm{C}$. This experiment should show: $1 /$ what time is

Table 1 Cell viability determined by a MTT assay for cells incubated with R6G-AuNPs and R6G in time intervals. Control cells were incubated with a pure medium

\begin{tabular}{llc}
\hline Incubation time $[\mathrm{h}]$ & \multicolumn{2}{c}{ Concentration of formazan [\% of control] } \\
\cline { 2 - 3 } & R6G-AuNPs & R6G \\
\hline 0.5 & 97 & 100 \\
1 & 79 & 100 \\
2 & 73 & 90 \\
4 & 77 & 90 \\
16 & 37 & 83 \\
Control & 100 & 100 \\
\hline
\end{tabular}

required to saturate the endothelial cells by the dye, $2 /$ differences in kinetics of the uptake, consequently in the membrane transport, between conjugated and nonconjugated molecules of the dye, and finally $3 /$ the contribution of the active (involving cellular energy) and passive (a diffusive process) mechanism into the membrane transport of the endothelium. An overall summary of the kinetics of the uptake of non-conjugated and conjugated R6G molecules for the EA.hy926 cells is given in Fig. 1. During uptake of the dye, the fluorescence rises quite quickly on the timescale of $30 \mathrm{~min}$ in comparison to the control group indicating the presence of rhodamine $6 \mathrm{G}$ in the intracellular compartments of endothelial cells, and then the intensity of fluorescence emission for R6G continues to grow up to $2 \mathrm{~h}$. With a longer than $2 \mathrm{~h}$ incubation time, it is maintained at a relatively similar level indicating that saturation of the cells by the dye takes place within $2 \mathrm{~h}$. In terms of the export of the Raman reporter from the endothelial cells, a very slow decay process can occur after $16 \mathrm{~h}$ as an insignificant fluorescence decrease is found for this incubation time. Moreover, the fluorescence emission is very similar for both R6G and R6G-AuNPs on the whole timescale used in our protocol. This undoubtedly confirms the intracellular transport of a similar amount of the dye regardless of its binding to the gold nanoparticles in the medium. However, these data do not indicate whether the R6G-AuNPs or the bare R6G molecules are transported through the cellular membrane since the technique is not sensitive to the metallic SERS support. This will be confirmed by SERS mapping in the section below.

To assess what mechanism, i.e. cellular chemical-mechanical energy or a simple diffusion process, plays a dominant role in the uptake of the dye/nanoparticles, the fluorescence was measured for cell cultures at 25 and $4{ }^{\circ} \mathrm{C}$ (Fig. S4, ESM). Unfortunately, endothelial cells are very sensitive to the temperature stress and after $1 \mathrm{~h}$ the cellular necrosis occurred at room temperature. For cells incubated at $4{ }^{\circ} \mathrm{C}$ we were able to carry out further measurements even after $4 \mathrm{~h}$. At $4{ }^{\circ} \mathrm{C}$ all the cellular processes are slower, so the necrosis process appears later comparing to $25^{\circ} \mathrm{C}$.

The average intensity of fluorescence emission for cells incubated with R6G-AuNPs as well as with R6G for $30 \mathrm{~min}$ at 37,25 and $4{ }^{\circ} \mathrm{C}$ is $265 / 283,217 / 203$ and $221 /$ 221 [in relative fluorescence intensity], respectively for R6G-AuNPs/R6G, while the control group exhibits autofluorescence with the intensity of 193 regardless of temperature (Fig. 1 and Fig. S4, ESM). These results indicate that no clear trend can be found between the uptake of R6G and R6G-AuNPs at a given temperature in short time of the incubation. The fluorescence only slightly decreases along with inducing energy depletion. After the $1 \mathrm{~h}$ incubation, a higher concentration of R6G conjugated 


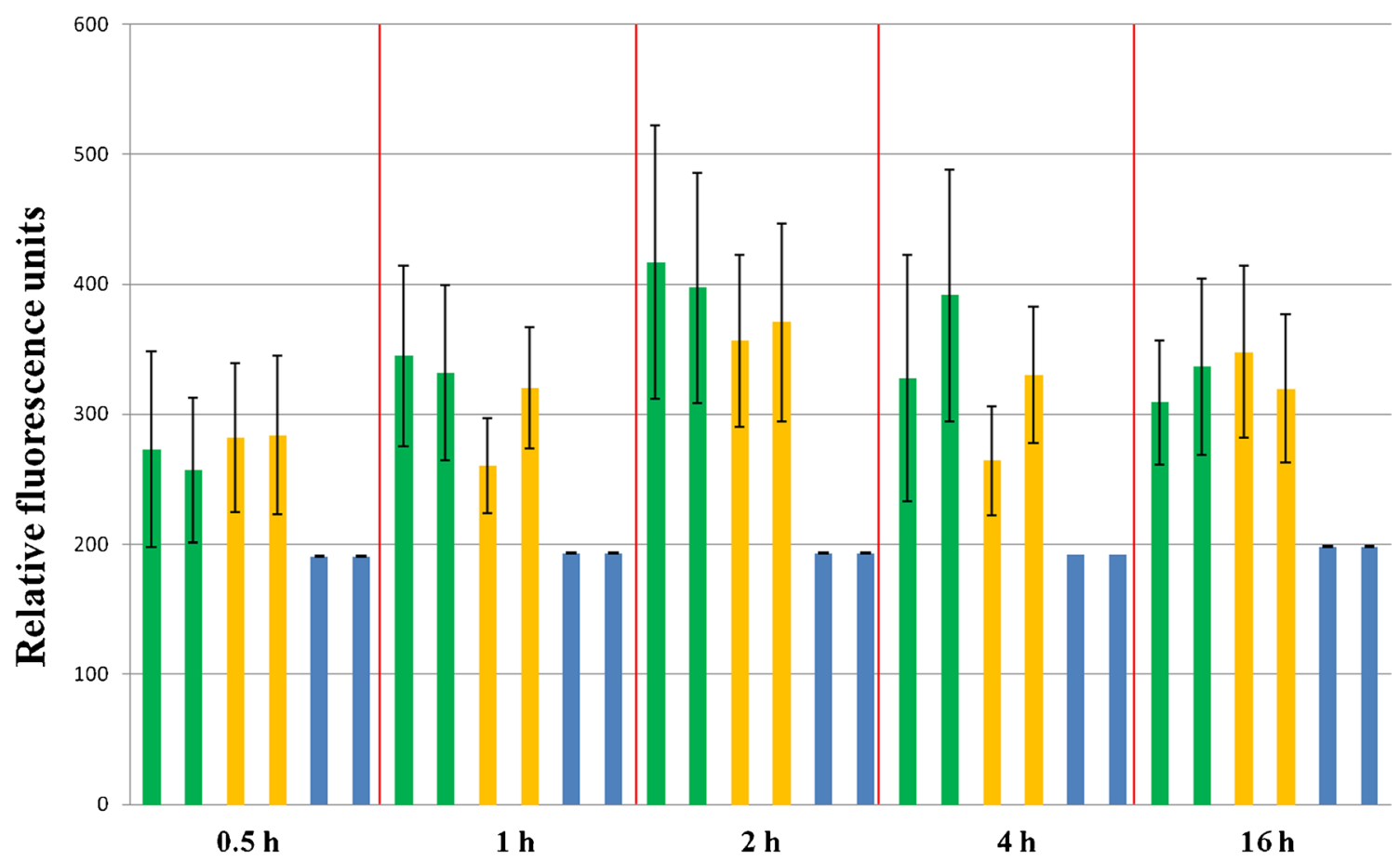

Fig. 1 The comparison of R6G-AuNPs (green) and R6G (yellow) uptake by EA.hy 926 endothelial cells for $0.5-16 \mathrm{~h} \mathrm{in} 37^{\circ} \mathrm{C}$. Control cells $($ blue $)$ were incubated in a label-free medium. The relative fluorescence intensities are provided along with standard deviation bars

to the gold nanoparticles than the bare $\mathrm{R} 6 \mathrm{G}$ is found in the endothelial cells incubated at 37 and $25{ }^{\circ} \mathrm{C}$ since relative fluorescence intensities are 338/290 and 260/ 238 , respectively. The results also suggest that a principal mechanism of cellular uptake of R6G-AuNPs but not of $\mathrm{R} 6 \mathrm{G}$ is an energy-dependent process of endocytosis. It is well known that the mechanism of endocytosis depends on the size of material and for NPs with a diameter smaller than $40 \mathrm{~nm}$, the docking of the NPs does not produce enough energy to completely wrap the NPs by the cell membrane $[26,27]$. In our work, the gold nanoparticles are of a $c a .40 \mathrm{~nm}$ diameter and they aggregate after mixing with the Raman reporter. Moreover, a very slight increase in fluorescence in comparison to the control group is observed for the cells incubated at $4{ }^{\circ} \mathrm{C}(212 /$ 226 for R6G-AuNPs/R6G). In this case, however, the fluorescence intensity has no obvious change compared to that observed for the 0.5 -h incubation but it increases after 2 h, c.f. Fig. S4B (ESM), indicating a slow diffusion process. At the present stage, it is very hard to clarify whether the difference in the fluorescence results between the cells with R6G-AuNPs and R6G is statistically significant (approx. 250-300 cells measured per well). The cellular delivery of R6G-AuNPs to the endothelium via active and passive mechanisms may occur simultaneously in our experiment leading to the saturation of the cells after $2-4 \mathrm{~h}$.

The 2D intracellular fluorescence measurements of cells with the use of a high-content screening automated microscope enable the quantitative analysis of a dye for a large cell population [28, 29], however, this technique does not determine an exact location of the dye molecules in the intracellular compartment. Figure 2 illustrates fluorescence image of the EA.hy 926 cells with the Hoechststained nuclei and yellow staining of R6G distributed within cytoplasm. It is rather impossible to asses from this image whether the R6G molecules are present inside the cells, or possibly they are simply attached to the membrane surface of the endothelial cells.

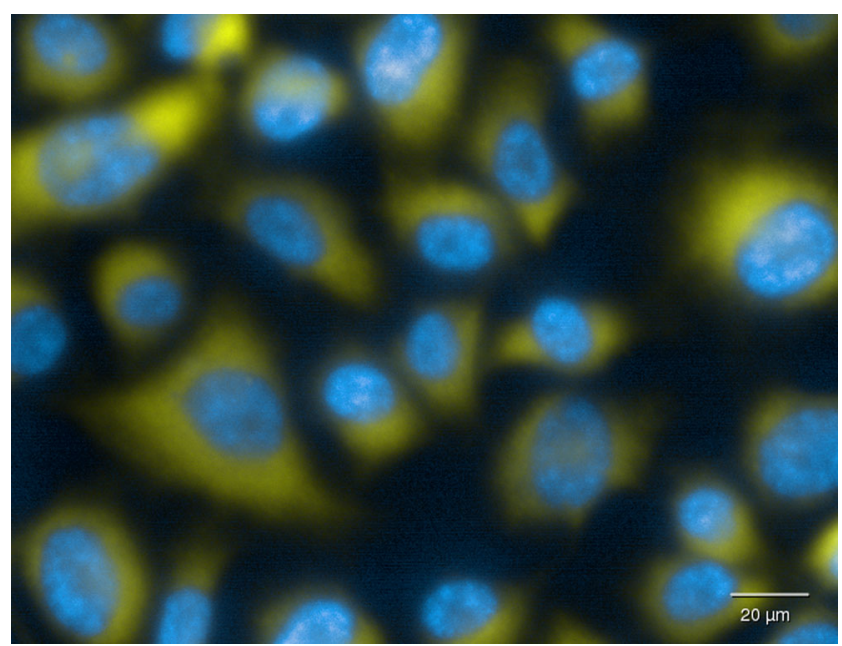

Fig. 2 The rhodamine $6 \mathrm{G}$ molecules detected in cytoplasm of EA.hy 926 endothelial cells by 2D fluorescence microscopy. Nuclei stained with Hoechst 33342 are blue, R6G is yellow 

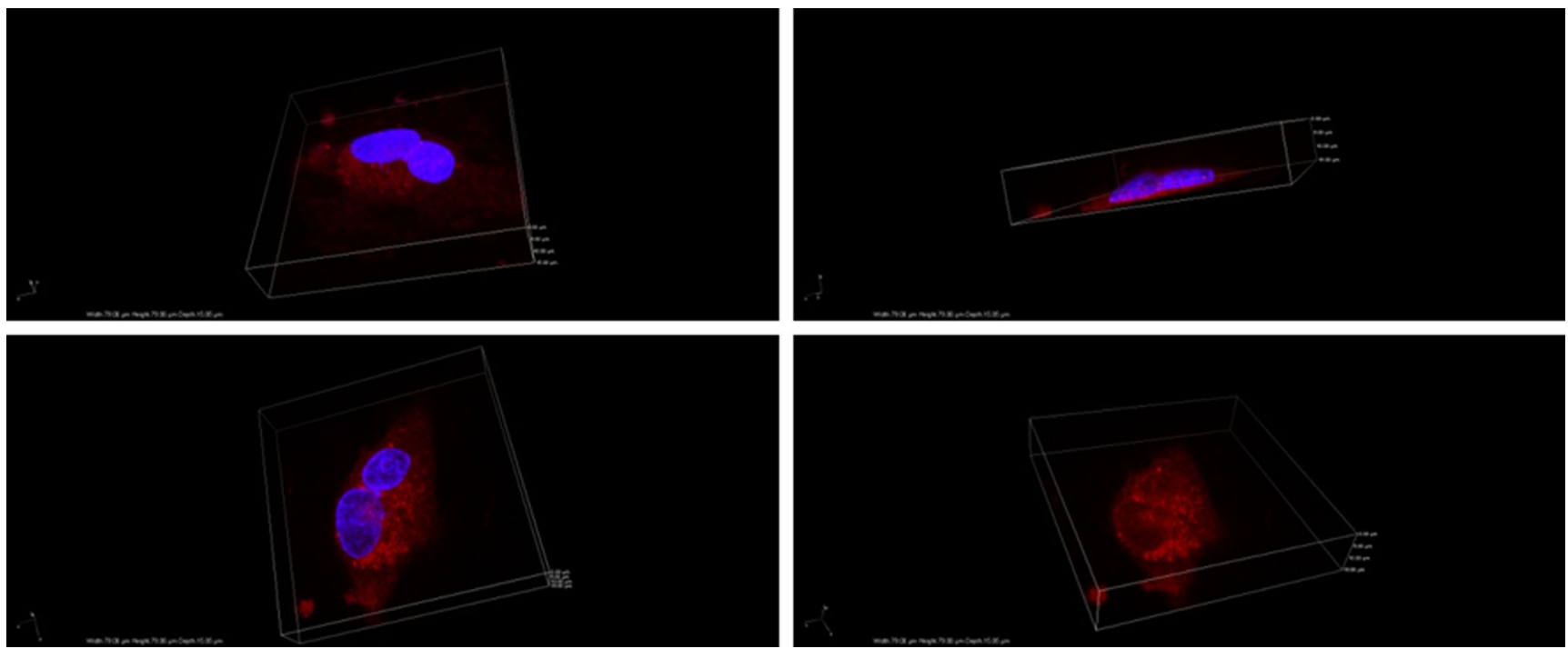

Fig. 3 3D confocal fluorescence images of endothelial cells incubated for $2 \mathrm{~h}$ with R6G-AuNPs. Blue: nuclei stained with Hoechst, red: rhodamine 6G. The right bottom image shows the distribution of R6G only

The location of R6G in the endothelial cells: 3D confocal fluorescence

3D confocal microscopy was employed to confirm the intracellular uptake of R6G-AuNPs by the endothelial cells. Figure 3 illustrates location of the nucleus surrounded by the dye spread throughout the cell without entering to the nucleus. The changes in the fluorescence intensity show that small aggregates of the Raman reporter dye are distributed unevenly in the cytoplasm but it is rather impossible to determine if they are localised in specific cell compartments.

Chemical information: SERS mapping of the endothelial cells

As shown above, the fluorescence provides information on the cellular distribution of rhodamine $6 \mathrm{G}$ but this technique does not differentiate the bare and conjugated dye so it is difficult to follow the presence/absence of R6G conjugated to AuNPs. To investigate this as well as to analyse potential chemical information gathered from molecular vibrations of biomolecules, surface-enhanced Raman mapping was performed for the endothelial cells incubated with R6G-AuNPs. The control groups were the cell cultures incubated in the label-free medium as well as in the medium containing R6G. For both groups, no Raman signal was recorded indicating that the use of the $633 \mathrm{~nm}$ laser excitation and short integration time $(0.05 \mathrm{~s})$ do not provide intracellular chemical information without a SERS support (data not shown). This has been also reported by Sathuluri et al. [30]. Figure 4 illustrates microscopic images of representative cells along with $\mathrm{K}$ means cluster maps and mean SERS spectra extracted from the corresponding classes. The SERS bands of rhodamine $6 \mathrm{G}$ are observed in the spectra recorded for the incubation time up to $2 \mathrm{~h}$. Their distributions inside the cells shown in the cluster maps implicates a decrease in the concentration of the R6G-AuNPs particles upon long incubation time and moving them towards the cell membrane, $c f$. pink traces in Fig. $4 \mathrm{a}-\mathrm{c}$. On the other hand, since the SERS spectra for each incubation time exhibit the presence of bands attributed to various biomolecules (discussed below), the R6G molecules must be deattached from the surface of the gold and then biomolecules present in the close vicinity of hot spots exhibit SERS signal. In our preliminary studies on probing cellular environment of the endothelium by the R6G-AuNPs label, we observed the SERS signal of the dye for the 2and 6-h incubation time while SERS bands originating from various biomolecules were observed up to $16 \mathrm{~h}$ [8]. This indicates that the conjugation of rhodamine $6 \mathrm{G}$ to nanoparticles can be stable up to a few hours. Salvati et al. have shown that after the uptake of nanoparticles containing a high concentration of a labile dye, the dye molecules are randomly spread across the intracellular space whereas the dye conjugated to nanoparticles is spatially localised [25]. A similar effect is observed in our experiment. As the 3D fluorescence image showed above, the labile R6G molecules fill up the cytoplasm, probably reaching the endosomes of the cell whereas the distribution of R6G-AuNPs in SERS maps is localised.

Apart from SERS bands of R6G, mean SERS spectra show the presence of marker bands of cell biomolecules assigned according to the literature, see Fig. $4[8,9,29,30]$. In the spectra of the cells incubated for $30 \mathrm{~min}$., bands attributed to proteins and lipids $\left(1,268,1,278,1,464,1,545 \mathrm{~cm}^{-1}\right)$ and the 
Fig. 4 SERS mean spectra extracted from cluster maps of endothelial cells incubated with R6G-AuNPs for $0.5 \mathrm{~h}(\mathbf{a}), 1 \mathrm{~h}(\mathbf{b})$, $2 \mathrm{~h}(\mathbf{c}), 4 \mathrm{~h}(\mathbf{d})$, and $16 \mathrm{~h}(\mathbf{e})$ along with a microphotography of a cell and a cluster map. Black trace in (c) represents a reference SERS spectrum of rhodamine $6 \mathrm{G}$. The colours of spectra correspond to the colours of classes in the cluster map

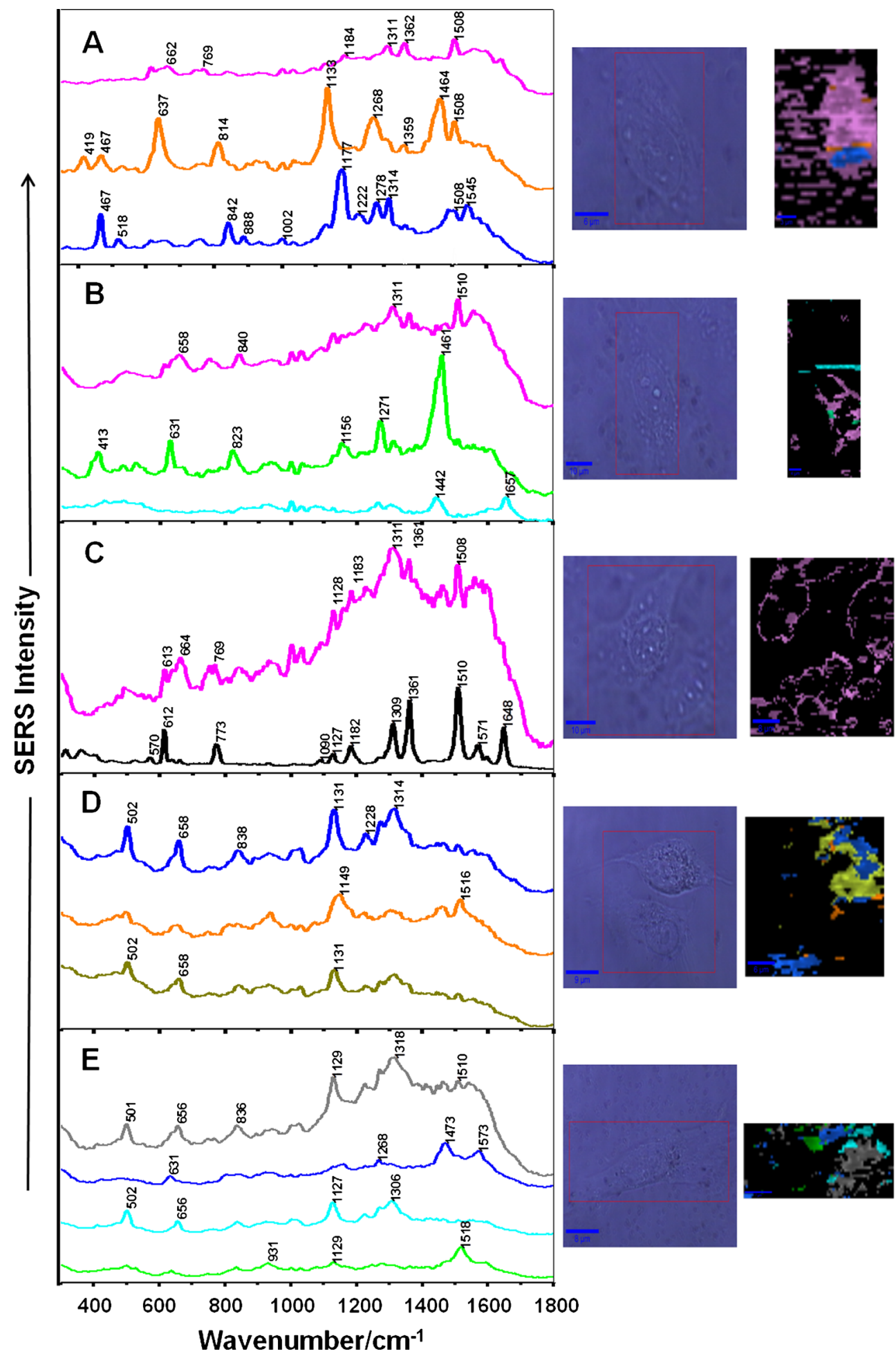

phosphate groups $\left(419,814 \mathrm{~cm}^{-1}\right)$ are found (Fig. 4a). After the 1-h incubation, protein and lipid bands are still observed (at 1,271, 1,442, 1,461, 1,657 $\mathrm{cm}^{-1}$ ) along with Raman markers for tyrosine $\left(631,823 \mathrm{~cm}^{-1}\right)$ and the $\mathrm{C}-\mathrm{C}$ and $\mathrm{C}-\mathrm{N}$ stretching vibrations $\left(1,156 \mathrm{~cm}^{-1}\right)$, c.f. Fig. $4 \mathrm{~b}$. In turn, a few bands originating from Cys $\left(664 \mathrm{~cm}^{-1}\right), \operatorname{Trp}\left(760 \mathrm{~cm}^{-1}\right)$ and
Phe $\left(1,001\right.$ and $\left.1,021 \mathrm{~cm}^{-1}\right)$ appear in SERS spectrum of the cells upon the 2-h incubation with R6G-AuNPs but this spectrum is mainly dominated by the R6G signal (Fig. 4c). For the 4 and 16-h incubation (Fig. 4d, e), we identified bands of amino acid residues such as cysteine $\left(502 \mathrm{~cm}^{-1}\right)$, tyrosine $\left(658,838 \mathrm{~cm}^{-1}\right)$, proline (931 and ca. $\left.1,130 \mathrm{~cm}^{-1}\right)$ and 
purines/pyridines $\left(1,228,1,268,1,314,1,516 \mathrm{~cm}^{-1}\right)$. Summarizing, the SERS label containing R6G as a Raman reporter enables sensitive and locally confined chemical probing of intracellular environment indicating mainly the presence of amino acid residues and moieties of nucleic acids.

\section{Conclusion}

The work demonstrates a detailed and multiparametric approach to assess the interactions of the endothelial cells with the gold nanoparticles immobilized with the Raman reporter-rhodamine 6G. Firstly, we show that the designed particles can interfere with normal cellular homeostasis within $4 \mathrm{~h}$, leading to substantial toxic effect after $16 \mathrm{~h}$. This suggests that although a non-toxic concentration of AuNPs is used in the experiment, their conjugation with a Raman reporter can induce cytotoxic mechanism in the endothelium especially during the long-time incubation, further emphasizing the importance of evaluating the toxic effects of nanomaterials on cultured cells. Then, we employ the chosen nanoparticles to study the cellular uptake behaviour of R6G-AuNPs by means of 2D and 3D fluorescence and SERS spectroscopy. We reveal that the uptake of the dye as well as the dye-Au support is a relatively fast process in that the cell is saturated by both species within $2 \mathrm{~h}$ and they are accumulated in the cytoplasm without an indication of a specific cellular compartment. The current work suggests that the mechanism of the cellular uptake is energy-dependent endocytosis. However, specific pathways of such a mechanism must be studied separately. In turn, intracellular SERS imaging provides the strong and stable signal of cellular components of the endothelium exhibiting the presence of amino acid residues and bases of nucleic acids. The comparison of both SERS and fluorescence imaging clearly shows that the Raman reporter is de-attached from the surface of SERS substrate in the intracellular environment already after $0.5 \mathrm{~h}$ of the incubation since SERS signal of biomolecules is observed. This may implicate a real-time process of exchanging adsorbates in the local optical field of the metal nanoaggregates and/or rearrangement of gold nanoaggregates in a way in which SERS hot spots are formed around other molecules than the dye. In summary, our studies also demonstrate the strength of a multimethodological approach for in vivo cell labelling applications and may provide hints to establish a reference protocol for further studies investigating interactions between cells and nanocarries.

Acknowledgments This work was supported by National Center of Science (DEC-2013/08/A/ST4/00308 and DEC-2012/05/N/ST4/00218) and by the European Union from the resources of the European Regional
Development Fund under the Innovative Economy Programme (grant coordinated by JCET-UJ, No POIG.01.01.02-00-069/09). We also thank Dr M. Ocwieja from the Jerzy Haber Institute of Catalysis and Surface Chemistry (Polish Academy of Science in Krakow) for AFM measurements and Msc. Eng. A. Szpak (Faculty of Chemistry, Jagiellonian University in Krakow) for DLS measurements. A.J. thanks Prof. H.J. Byrne from the Focas Research Institute in Dublin for the creative discussion. The research was carried out with the equipment purchased thanks to the financial support of the European Regional Development Fund in the framework of the Polish Innovation Economy Operational Program (Contract POIG.02.01.00-12-023/08).

Open Access This article is distributed under the terms of the Creative Commons Attribution License which permits any use, distribution, and reproduction in any medium, provided the original author(s) and the source are credited.

\section{References}

1. Auchinvole CAR, Richardson P, McGuinnes C, Mallikarjun V, Donaldson K, McNab H, Campbell CJ (2012) Monitoring intracellular redox potential changes using SERS nanosensors. ACS Nano 6: 888

2. Leopold N, Baena JR, Bolboaca M, Cozar O, Kiefer W, Lendl B (2004) Raman, IR, and surface-enhanced Raman spectroscopy of papaverine. An automated setup for in situ synthesis of the silver substrate and recording of the SER spectra. Vib Spectrosc 36:47

3. Culha M, Cullum B, Lavrik N, Klutse CK (2012) Surface-enhanced Raman scattering as an emerging characterization and detection technique. J Nanotechnol 12:1

4. Jaworska A, Malek K, Marzec KM, Baranska M (2012) Nicotinamide and trigonelline studied with surface-enhanced FTRaman spectroscopy. Vib Spectrosc 63:469

5. Kneipp J, Kneipp H, Kneipp K (2008) SERS - a single-molecule and nanoscale tool for bioanalytics. Chem Soc Rev 37:1052

6. Guo X, Guo Z, Jin Y, Liu Z, Zhang W, Huang D (2012) Silver-gold core-shell nanoparticles containing methylene blue as SERS labels for probing and imaging of live cells. Microchim Acta 178:229

7. Kneipp K, Haka AS, Kneipp H, Badizadegan K, Yoshizawa N, Boone C, Shafer-Peltier KE, Motz JT, Dasari RR, Feld MS (2002) Surface-enhanced Raman spectroscopy in single living cells using gold nanoparticles. Appl Spectrosc 56(2):150

8. Jaworska A, Malek K, Kachamakova-Trojanowska N, Chlopicki S, Baranska M (2013) The uptake of gold nanoparticles by endothelial cells studied by surface-enhanced Raman spectroscopy. Biomed Spectrosc Imaging 2:183

9. Malek K, Jaworska A, Krala P, Kachamakova-Trojanowska N, Baranska M (2013) Imaging of macrophages by Surface Enhanced Raman Spectroscopy (SERS). Biomed Spectrosc Imaging 2:349

10. Schlucker S (2011) Surface enhanced Raman spectroscopy. WILEYVCH Verlag GmbH \& Co. KgaA, Germany

11. Kneipp J, Kneipp H, Rajadurai A, Redmond RW, Kneipp K (2009) Optical probing and imaging of live cells using SERS labels. J Raman Spectrosc 40:1

12. Halbhuber KJ, Konig K (2003) Modern laser scanning microscopy in biology, biotechnology and medicine. Ann Anat 185:1

13. Pygall SR, Whetstone J, Timmins P, Melia CD (2007) Pharmaceutical applications of confocal laser scanning microscopy: the physical characterisation of pharmaceutical systems. Adv Drug Deliv Rev 59(14):1434

14. Fischer RS, Wu Y, Kanchanawong P, Shroff H, Waterman CM (2011) Microscopy in 3D: a biologist's toolbox. Trends Cell Biol 21(12):682 
15. Conchello JA, Lichtman JW (2005) Optical sectioning microscopy. Nat Methods 2(12):920

16. Foldes-Papp Z, Demel U, Tilz GP (2003) Laser scanning confocal fluorescence microscopy: an overview. Int Immunopharmacol 3:1715

17. Félétou M (2011) Multiple functions of the endothelial cells. In: The endothelium part 1: multiple functions of the endothelial cells - focus on endothelium-derived vasoactive mediators. Morgan \& Claypool Life Sciences, San Rafael

18. Wnuczko K, Szczepanski M (2007) Endothelium - characteristics and functions. Pol Merk Lek XXIII(133):60

19. Frens G (1973) Controlled nucleation for the regulation of the particle size in monodisperse gold suspensions. Nat Phys Sci 241:20

20. Edgell C, McDonald C, Graham J (1983) Permanent cell line expressing human factor VIII-related antigen established by hybridization. Proc Natl Acad Sci 80:3734

21. Otto F, Tsou KC (1985) A comparative study of DAPI, DIPI, and Hoechst 22358 and 33342 as chromosomal DNA stains. Stain Technol 60(1):7

22. Joseph V, Matschulat A, Polte J, Rolf S, Emmenrling F, Kneipp J (2011) SERS enhancement of gold nanospheres of defined size. J Raman Spectrose 42:1736

23. Asare N, Instanes C, Sandberg WJ, Refsnes M, Schwarze P, Kruszewski M, Brunborg G (2012) Cytotoxic and genotoxic effects of silver nanoparticles in testicular cells. Toxicology 291(1-3):65
24. Soenen SJ, Manshian B, Montenegro JM, Amin F, Meermann B, Thiron T, Cornelissen M, Vanhaecke F, Doak S, Parak WJ, De Smedt S, Braeckmans K (2012) Cytotoxic effects of gold nanoparticles: a multiparametric study. ACS Nano 6(7):5767

25. Salvati A, Aberg C, dos Santos T, Varela J, Pinto P, Lynch I, Dawson KA (2011) Experimental and theoretical comparison of intracellular import of polymeric nanoparticles and small molecules: toward models of uptake kinetics. Nanomedicine 7(6):818

26. Wang SH, Lee Ch W, Chiou A, Wei PK (2010) Size-dependent endocytosis of gold nanoparticles studied by three-dimentional mapping of plasmonic scattering images. J Nanobiotechnol 8:33

27. Huang J, Zong C, Shen H, Liu M, Chen B, Ren B, Zhang Z (2012) Mechanism of cellular uptake of grapheme oxide studied by surfaceenhanced Raman spectroscopy. Small 8:2577

28. Xu Z, Huang X, Dong C, Ren J (2014) Fluorescence correlation spectroscopy of gold nanoparticles, and its application to an aptamer-based homogeneous thrombin assay. Microchim Acta $181: 723$

29. Kneipp J, Kneipp H, McLaughlin M, Brown D, Kneipp K (2006) In vivo molecular probing of cellular compartments with gold nanoparticles and nanoaggregates. Nano Lett 6(10):2225

30. Sathuluri RR, Yoshikawa H, Shimizu E, Saito M, Tamiya E (2011) Gold nanoparticle-based surface-enhanced Raman scattering for noninvasive molecular probing of embryonic stem cell differentiation. PLoS One 6(8):e22802 